\title{
Transforming Growth Factor
}

National Cancer Institute

\section{Source}

National Cancer Institute. Transforming Growth Factor. NCI Thesaurus. Code C20453.

Transforming Growth Factors are a family of extracellular signaling peptides involved in cell growth stimulation. Found in hematopoietic tissue, various T GF-beta subtypes stimulate wound healing and may inhibit lymphopoiesis and myelopoiesis. TGF-alpha binds to EGFR and stimulates endothelial cell growth. 